\title{
CHRONICLE
}

\section{Congratulations to the Laureates of the State Prize of Ukraine in the field of science and technology for 2019}

By presidential decree No. 4 of January 13, 2020, a team of researchers comprising V. A. Chebanov, S. M. Desenko, N. A. Lyapunov (Scientific and Technological Complex "Institute for Single Crystals" of the National Academy of Sciences of Ukraine, Kharkiv), V. S. Brovarets (V. P. Kukhar Institute of Bioorganic Chemistry and Petrochemistry of the National Academy of Sciences of Ukraine, Kyiv), M. V. Vovk (Institute of Organic Chemistry of the National Academy of Sciences of Ukraine, Kyiv), V. V. Lipson (V. Ya. Danilevsky Institute of Problems of Endocrine Pathology of the National Academy of Medical Sciences of Ukraine, Kharkiv), R. B. Lesik (Lviv National Medical University, Lviv), N. D. Obushak (Ivan Franko National University of Lviv, Lviv) was awarded the State Prize of Ukraine in the field of science and technology for 2019 for the work "Highly selective methods for the synthesis of heterocyclic compounds for the development of components of functional materials and the creation of new drugs". The indicated work was presented by the State Scientific Institution "Scientific and Technological Complex "Institute for Single Crystals" of the National Academy of Sciences of Ukraine".

The team members proposed a new concept of controlled organic synthesis, discovered the latest and substantially developed the known methods of highly selective design of new types of heterocyclic systems with powerful potential for creating components of functional materials, as well as for the discovery of bioactive substances. The fundamental principles of organic chemistry were developed, which relate to the structure of organic compounds, their reactivity, and directed functionalization. A number of new environmentally attractive, preparatively available, and effective reagents and catalysts have been introduced in the practice of heterocyclic chemistry. Based on the developed methods of heterocyclic synthesis, new dyes, complexing agents, fungicides and herbicides, components of photoactive and radiochromic schemes and functional materials for organic electronics were obtained.

Using methods of pharmacological screening, in silico studies of the structure-action correlation, and in vitro and in vivo biological tests, priority types of activity for the classes of studied heterocycles have been outlined and a
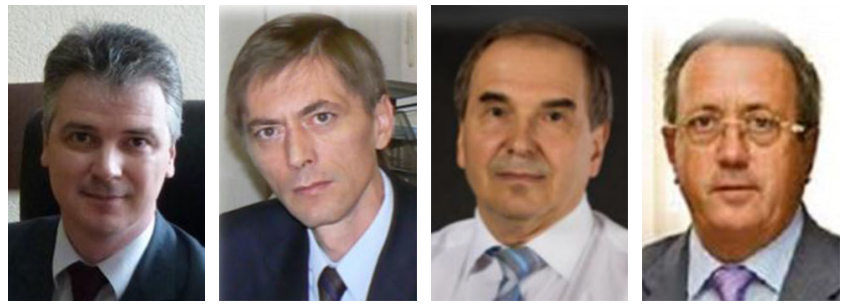

V. A. Chebanov S. M. Desenko N. A. Lyapunov V. S. Brovarets

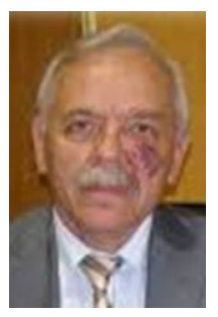

M. V. Vovk

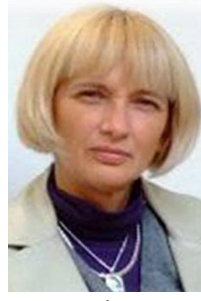

V. V. Lipson

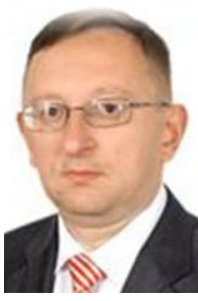

R. B. Lesik

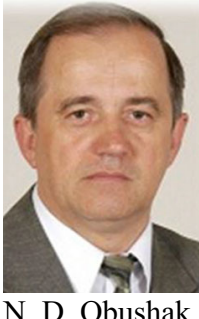

N. D. Obushak wide range of their biological and medicinal properties has been experimentally established against low toxicometric parameters. Lead compounds were identified for further optimization, in-depth studies, and directed synthesis of new biologically active molecules as potential drugs.

Fundamental research has been carried out on the creation of various types of carriers, which are systems with a liquid dispersed medium and have predetermined physicochemical properties. The mechanisms of physical stabilization of carriers are determined and their effect on the functional properties of drugs was investigated. A system of regulatory documents has been developed that regulates the circulation of drugs and 75 new forms of drugs have been created that have been introduced into industrial production at pharmaceutical enterprises in Ukraine.

Laureates V. A. Chebanov and S. M. Desenko are members of the editorial board, and V. S. Brovarets, M. V. Vovk, V. V. Lipson, and N. D. Obushak are active authors of the journal "Chemistry of Heterocyclic Compounds". The editorial board of the journal "Chemistry of Heterocyclic Compounds" congratulates fellow laureates on the State Prize of Ukraine in the field of science and technology and wishes them new creative successes and scientific achievements.

Professor, Dr. Chem. Sci. Yu. G. Shermolovich, Deputy Director of the Institute of Organic Chemistry of the National Academy of Sciences of Ukraine 